\title{
LA ECONOMÍA Y LA NATURALEZA HUMANA
}

\author{
Carlos Arturo Dávila Rojas
}

\section{INTRODUCGIÓN}

Muchas ciencias, especialmente las denominadas sociales, se están redefiniendo a partir del conocimiento de la naturaleza humana, entendida como la esencia misma del Hombre $e^{1}$ y no simplemente una particularidad de ésta; es decir, la raíz de lo que es el hombre en sí. En el caso de la Ciencia Económica, el estudio de todas las formas de consumo, producción e intercambio que son acciones o conductas económicas -naturales e instintivas- y significan asignaciones racionales de recursos, constituyen su ámbito positivo: lo que es. Pero, como ciencia, no se limita a observar las consecuencias del accionar económico natural de los individuos; sino también realiza prescripciones para optimizar sus resultados haciéndolos paretianos, las que constituyen su ámbito normativo: lo que debe ser.

\section{Características básicas de la NATURALEZA O CONDICIÓN HUMANA}

En base a los conocimientos más importantes de la reflexión filosófica y científica existente sobre la naturaleza humana, presentamos algunas de las características más importantes de tal condición; antes de la formación ética o marco moral y de la creación del Estado institucional en base a normas $y$ autoridades.

\section{a. No somos buenos ni malos por naturaleza}

El término bueno se usa en la acepción adjetiva de bondad que significa calidad de bueno. Natural inclinación a hacer el bien. En cambio, el término malo se usa en la acepción adjetiva de maldad que significa calidad de malo, acción mala. (Larousse: Diccionario Enciclopédico, 1998). Los individuos, de manera recurrente, despliegan acciones o decisiones evaluadas como buenas o malas. Normalmente, actuar de una u otra forma estará relacionado a las reacciones u objetivos necesarios o en juego, respectivamente.

En la evaluación interna o externa de una acción buena o mala es la percepción del propio individuo lo que cuenta; o sea, razones y virtudes son sólo individuales. Los actos de bondad y maldad dependerán de lo que más impacte en el bienestar u objetivos de la persona, evaluada individualmente.

\section{b. No somos altruistas por naturaleza}

La expresión altruista se utiliza significando la acción beneficente, caritativa o filantrópica que intenta beneficiar o apoyar la empresa o causa del prójimo, sin esperar acción solidaria o recíproca. También significa "... cuidado desinteresado al bien ajeno, aún a costa del propio y fundado en una moral puramente natural" (Ibid.)

La forma más extendida de esta conducta es el generalizado desinterés que observan los individuos por las causas ajenas. También, abona a esta conducta el desprendimiento esporádico de muy pequeños recursos propios que unas personas otorgan a otros que necesitan o piden ayuda: caso limosnas de caridad. 
No debe confundirse con altruismo a las extendidas acciones de cooperación y reciprocidad que realizan los individuos en sus redes afectivas y cercanas; familiares, laborales, amicales, etc. Tales acciones están marcadas por su carácter solidario, que demandan reciprocidad, compatibles con la extendida tradición de hoy por ti, mañana por mí.

\section{c. Somos violentos por naturaleza.}

Tenemos propensión a la violencia como forma natural de resolver diferencias y de imponer nuestros intereses, siempre considerados superiores al de los otros. Buscamos y nos agrada pertenecer a cada vez mayores redes para competir con otros. Las redes sociales que conformamos o a las que nos adherimos (familiares, políticas, religiosas, deportivas, etc.) tienen sentido sólo en su enfrentamiento, abierto o soterrado, con las otras. En estas redes buscamos y logramos la mayor identidad y reconocimiento posible. En ausencia de tales redes y competencia, el individuo desaparecería. Se afirma que el estado natural de la humanidad es la violencia, entendiéndose a la paz como el equilibrio alcanzado por las partes en conflicto.

\section{d. Somos racionales por naturaleza.}

El término racional se utiliza en su acepción de justificación beneficiosa de una acción o causa y de conformidad o equivalencia a la eficiencia. Los individuos logran o esperan de sus acciones y decisiones el mayor beneficio posible, respecto a sus costos. En ausencia de formación moral y de institucionalidad, normas, autoridades, etc., dichos costos y beneficios son básicamente privados, de corto plazo y sin mucha consideración por los costos sociales y las externalidades; especialmente negativas.

\section{e. Somos libres por naturaleza.}

Ejercemos la libertad por naturaleza. La noción de libertad debe entenderse como el poder inmanente al sujeto y definirse como la capacidad de decisión, autodeterminación o facultad natural del hombre para obrar de una manera u otra, o no obrar, y por la que es responsable de sus actos. Previo a su decisión, el individuo tiene alguna información; pero, la decisión última, la toma libremente.

\section{f. Somos egoístas o individualistas por naturaleza.}

Es la supremacía que ponen los individuos a su ego o yo. Es el deseo de reconocimiento y la búsqueda de vanagloria. Todos los individuos parecieran tener un sistema de ecuaciones en la que:

$$
\begin{aligned}
& \mathrm{YO}>\mathrm{TU}, \\
& \mathrm{MI}>\mathrm{TU} ; \Leftrightarrow \mathrm{YO}>\mathrm{MI}
\end{aligned}
$$

En Fukuyama ${ }^{2}$, encontramos que hay dos tipos de individualismo o autoestima: los isothimus y los megathimus. Los primeros, son aquellos con niveles normales de autoestima; en tanto que los segundos tienen niveles de autoestima más intensos y son los principales actores de la historia, entendida como transcurso de acontecimientos. Casi toda la lógica de las acciones y decisiones del individuo está regida por su nivel de egoísmo; existiendo fuerte correlación entre el logro de deseos y el individualismo.

\section{g. Somos políticos y transaccionales por naturaleza.}

Aunquenuestralógicanatural esindividualista, seríamos gregarios, con vida grupal o en sociedad, por necesidad; buscando posiciones de representación y, en su contexto, para resolver nuestras necesidades, especialmente nuestros deseos, estamos obligados a tomar decisiones bajo interdependencia. Tenemos la capacidad de establecer redes, relaciones de cooperación, reciprocidad y transacciones, en base a la interacción. Estableciendo relaciones de confianza buscar y siempre procurando resultados que nos sean beneficiosos.

\section{LA GONDUCTa ECONÓMICA NATURAL DE LOS INDIVIDUOS}

Se identifica al consumo, la producción y el intercambio, en todas sus formas, como los ámbitos de asignación racional de recursos escasos que hacen los individuos. Estas acciones o actividades se realizan de manera natural e instintiva; se habrían iniciado con la aparición misma de la especie humana en la faz de la tierra; por lo que forman parte de la natural conducta humana. La economía, en su ámbito positivo, estudia la conducta humana que usa o asigna recursos escasos, entre

2. Fukuyama, Francis. El Fin de la Historia y el Último Hombre. 
opciones alternativas; asociados a la satisfacción de sus necesidades y deseos que son ilimitados. La microeconomía es la modelación teórica de la conducta económica natural de la especie humana.

La noción de recursos está referida a todo bien, servicio, factor productivo, tecnología, dimensión -como tiempo y espacio, etc.- sujeto a asignación. La noción de escasez es relativa, asociada al carácter ilimitado de las necesidades y deseos de los individuos. Las necesidades son las sensaciones, inquietudes e imperativos humanos que requieren ser satisfechos o resueltos a través del uso o consumo de ciertos bienes y servicios. Las necesidades biológicas son consideradas primarias, tales como la alimentación, vestimenta, salud y vivienda; las de urgente e impostergable satisfacción. Asociado a la modernización de las sociedades, la noción de necesidades se ha ampliado a las de carácter secundario, como la educación, cultura, ocio, etc. Los deseos, relacionados al carácter egoísta de los individuos, están referidos a la normal propensión de los individuos a la trascendencia. La búsqueda de éxito, prestigio, vanagloria y reconocimiento parecieran ser las razones últimas y orientadoras del quehacer humano. Sólo son perseguidos y alcanzados en condiciones de ejercicio pleno de la libertad que es otra condición natural de los individuos. La historia, entendida como el transcurso de la humanidad y la construcción de la sociedad, es abierta; constituye el escenario de la búsqueda de reconocimiento de sus individuos libres. La noción de asignación racional es equivalente a asignación eficiente, en el entendido en que los beneficios o resultados de la asignación deben ser superiores a los costos. El balance de costos y beneficios se hace en términos marginales y debe ser integral; o sea, incluir los valores privados y sociales. La noción de opciones alternativas significa que la asignación de recursos tiene varias opciones o costo de oportunidad, asociada a la libertad de elección. Sólo comparando las opciones se puede elegir o conocer la mejor. Esta es la noción de maximización.

\section{¿QUÉ JUSTIFICA LA EXISTENCIA DE LA CiENCIA ECONÓMICA?}

El desarrollo de las ciencias, con sus métodos de modelar la realidad, no ocurre por motivación cultural ni cognitivismo puro. La existencia de

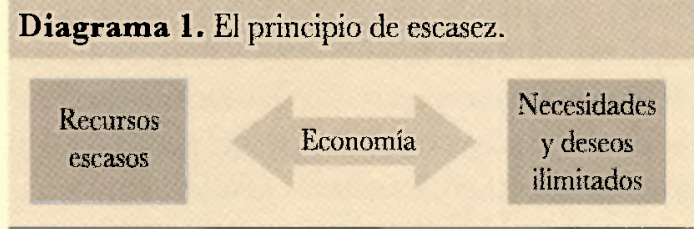

problemas $^{3}$ que la humanidad enfrenta y la necesidad de conocerlos y resolverlos es el motivo principal de su existencia. Se desarrolla a través de teorías construidas con métodos científicos. La ciencia y la profesión médica no tendrían razón de existir, si en la vida real, las enfermedades no fueran un problema.

Según el Principio de Escasez (Diagrama 1), los recursos son escasos o limitados; en tanto, que las necesidades y deseos de los individuos, son ilimitados. Este principio encierra el origen y la esencia de la Ciencia Económica.

Los problemas de qué, cómo y para quién producir no constituirían ningún problema si los recursos fueran ilimitados. No importaría que se fabrique demasiada cantidad de una determinada cosa ni que el trabajo y los materiales se combinen o no asociados a eficientes funciones de producción. Si no hubiese escasez de recursos; o si de manera natural fueran asignadas de manera paretiana; o si las necesidades y deseos humanos no fueran infinitos, no habrían problemas económicos; no serían necesarios la ciencia económica ni los economistas. Como todo el mundo podría tener tantos bienes como quisiera de cada cosa, no importaría el reparto de los ilimitados bienes entre los distintos individuos o grupos. Como lamentablemente los recursos no son ilimitados, entonces se los debe economizar usándolos racionalmente; pero si de manera natural los individuos asignaran los recursos racionalmente, ¿dónde estaría el problema?

\section{Resultados naturalmente RAGIONALES DE LAS ASIGNACIONES Y DECISIONES DE LOS INDIVIDUOS}

En condiciones normales, de manera natural, los individuos o grupos asignan recursos y toman decisiones racionales con beneficios superiores a sus costos, evaluados individualmente. Sin embargo, muchas de sus asignaciones y decisiones generan un perdedor. El hurto, los 
juegos de azar y una infinidad de acciones $y$ asignaciones delictuosas o ineficientes, evaluadas social y económicamente, pueden ser considerados racionales desde el balance privado; pero, en tanto generan sólo transferencias o pérdidas a terceros o contrapartes no pueden ser considerados como hechos o decisiones económicas en el sentido normativo de la Teoría o Ciencia Económica porque el tamaño de la torta (riqueza total) es el mismo; o se reduce o sólo genera redistribuciones.

\section{La Racionalidad Paretiana}

El término paretiano se deriva de las nociones de Óptimo o Equilibrio de Pareto; fue establecido en memoria del economista y sociólogo italiano Vilfredo Pareto. Veamos el siguiente Gráfico $\mathrm{N}^{\circ} 1$ y su explicación para entenderlo mejor.

Se denomina utilidad a la satisfacción que reporta a la persona el consumo de una determinada combinación de bienes, medida cardinalmente. Si obtiene más bienes, su utilidad aumenta. La Frontera de Posibilidades de Utilidad, FPU, representa el nivel máximo de utilidad que puede alcanzar un consumidor o grupo, dado el nivel de utilidad del otro consumidor o grupo y viceversa. La frontera de posibilidades de utilidad mide todas las asignaciones eficientes u óptimas en el Sentido de Pareto, puntos $\boldsymbol{A}, \boldsymbol{B}, \boldsymbol{C}, \boldsymbol{D}, \boldsymbol{E}$. Cada equilibrio óptimo representa eficiencias del consumo, la producción y el intercambio, dado los recursos y la tecnología, dentro de reglas de juego competitivas. Una economía es eficiente en el sentido de Pareto sí y solo sí está operando a lo largo de la FPU. Hay eficiencia o equilibrio en el sentido de Pareto si no es posible mejorar el bienestar de una persona sin empeorar el de otra. Es decir, no podemos aumentar la utilidad del Grupo I sin reducir la utilidad del Grupo II. Si una economía es eficiente en el sentido de Pareto debe encontrarse a lo largo de la FPU. Si se situara en un punto por debajo de la frontera, como el punto $\boldsymbol{x}$ del Gráfico $\mathrm{N}^{\circ}$ 1, sería posible aumentar la utilidad del Grupo I, cambio desde $\boldsymbol{x}$ a $\boldsymbol{z}$, sin reducir la utilidad del Grupo II; o viceversa, cambio desde $\boldsymbol{x}$ a $\boldsymbol{y}$ o aumentar la utilidad de los dos grupos, cambio desde $\boldsymbol{x}$ a $\boldsymbol{w}$. A esos cambios se les denomina Movimientos en Sentido Paretiano; nadie pierde, aunque no llegaran al óptimo de Pareto. Dado un punto de partida ineficiente y desigual, como el punto $\boldsymbol{x}$. el mercado competitivo

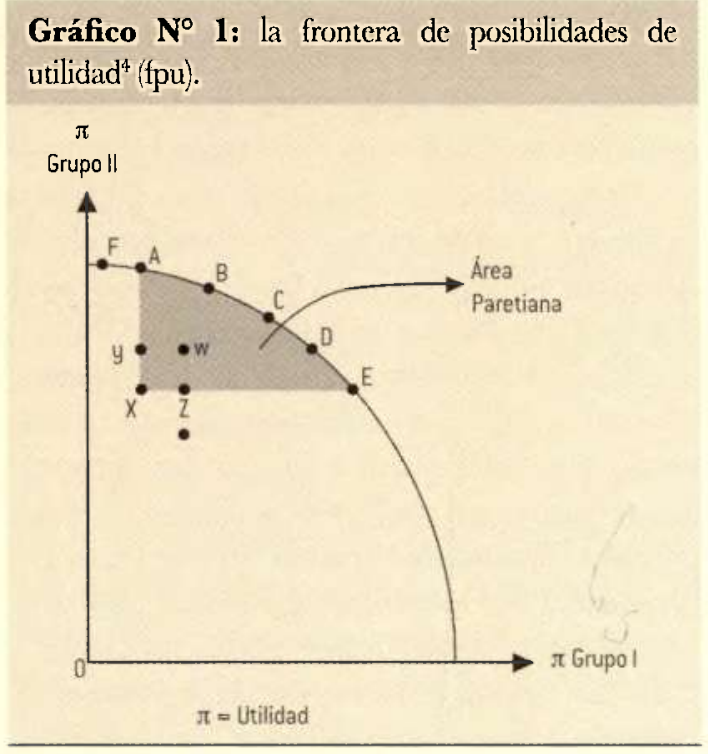

garantiza que se llegue a la eficiencia paretiana, o sea de $\boldsymbol{x}$ hacia $\boldsymbol{A}, \boldsymbol{B}, \boldsymbol{C}, \boldsymbol{D} \circ \boldsymbol{E}$. El cambio de $\boldsymbol{x}$ a $\boldsymbol{m}$ constituye una decisión o asignación de recursos no paretiano que genera un perdedor. Igualmente, un cambio desde puntos ineficientes, como $\boldsymbol{x}$ o $\boldsymbol{y}$ hacia F significa que se llegó a un equilibro óptimo, con resultado no paretiano, donde pierde el Grupo $I$. Si los mercados funcionan competitivamente es difícil que ocurran resultados no paretianos.

La noción de resultado paretiano está referida a una asignación racional de recursos que no genera un perdedor. Es decir, no valen los resultados de suma cero ni negativos. En un escenario competitivo el emprendimiento productivo, el intercambio y el consumo, son resultados paretianos; o sea eficientes, y no generan un perdedor. Las acciones humanas que generan transferencias y no crean riqueza neta, aun cuando pueden ser racionales -desde el balance de quienes se benefician- no pueden ser consideradas eficientes o válidas ni social ni económicamente. El stock de riqueza privada y social se mantiene o se reduce provocando permanente inseguridad, incertidumbre y pobreza. Todo esto hace inviable la vida social y desincentiva la capacidad de creación neta de valor. Por lo tanto, no todo lo que es racional es paretiano. En el ámbito normativo social de la Ciencia Económica, será esta última condición la que determine la validez socioeconómica o no de las decisiones y asignaciones de recursos de los individuos. La prohibición o condicionamiento legal de muchas

4. Conceptos desarrollados en base a Stiglitz, J. La Economia del Sector Público. (2003, pảg. 76) 
acciones humanas, beneficiosas privadamente, pero que generan perdedores, tiene su explicación e inspiración en el criterio de resultado paretiano. Se hará necesario construir una institucionalidad económica competitiva para garantizar asignaciones racionales y paretianas de los recursos escasos, en observancia al ámbito normativo de la ciencia económica. En aras de preservar la sociedad y su desarrollo, sólo deben validarse aquellas acciones o decisiones humanas que sean creadoras netas de riqueza económica y social; es decir, las que garanticen un crecimiento económico sostenido y fortalezcan la convivencia social.

¿Qué alternativas u opciones procesó la humanidad para garantizar que las acciones humanas socioeconómicas tuvieran resultados paretianos? ¿Era necesario cambiar la naturaleza humana o sólo optimizarla?

\section{FormaGión ÉTICA O GONCIENCIA MORAL, ¿OPTIMIZANDO LA NATURALEZA HUMANA?}

Las consecuencias del accionar no paretiano de los individuos habría generado la necesidad de definir e identificar al bien y la verdad como los fines del hombre. Se trata de acciones de educación moral para preferir la bondad a la maldad, la tolerancia a la violencia, el altruismo al egoísmo, etc., y convertir estas preferencias en hábitos humanos alcanzados por medio de la libertad y la voluntad. La naturaleza humana se realiza y perfecciona mediante decisiones libres que nos hacen mejores porque desarrollan nuestras capacidades. La ética es algo intrínseco a la persona, a su educación y a su desarrollo natural. Sin ética no hay desarrollo personal ni espiritual. La naturaleza humana se perfecciona con los hábitos porque modifican al sujeto que los adquiere, modulando su conducta de una determinada manera, pero sin eliminar su naturaleza.

\section{a. Los formadores de la ética o convicciones morales}

Históricamente, el esfuerzo por educar e inculcar valores a los individuos se habría iniciado antes de la creación del Estado, en los ámbitos familiares y grupales de las primeras colectividades humanas, en las que ya había propiedad privada, familia y convicciones religiosas, como formadoras de conciencia. Es reconocido que la familia da formación de valores, especialmente en la niñez, y costumbres que moldean y definen su conducta y personalidad para el resto de su vida. En el caso de las iglesias, casi todas las religiones son veneradas con respeto por sus seguidores. De manera clásica, las actividades religiosas y de fe que imparten las iglesias son fuertes formadoras de valores morales; por lo que se hizo conveniente garantizar la libertad de creencia y separar al Estado de la Iglesia.

\section{b. Limites optimizadores del marco moral o la ética}

Debido al contundente y recurrente accionar libre, racional y egoísta de los individuos, la eficacia optimizadora de la educación moral fue limitada; lo que hizo necesario recurrir a formas coactivas de evitar resultados no paretianos e injustos; procediéndose a inventar o crear el Estado.

\section{Origen del Estado}

La creación del Estado como desarrollo y el establecimiento de una buena institucionalidad -autoridades y normas de observancia obligadasque sólo valide los actos y decisiones paretianos han permitido y están permitiendo la optimización del accionar natural de los individuos; haciendo de la construcción social un proceso abierto, libre y creador de riqueza. Aunque el Estado fue un gran invento para organizar la convivencia social, garantizar la vigencia de la especie humana y el desarrollo de la sociedad, históricamente muchas veces los gobernantes lo han utilizado para acciones socialmente destructivas o para proteger intereses de grupo. Asociado a su creación, hubo y aún hay un debate para definir su carácter mismo.

\section{a. Estado: ¿Amo o servidor?}

Para los seguidores de Thomas Hobbes ${ }^{5}$, el Estado se ha creado para constituirse como el amo de los habitantes, en nombre de quienes y sobre quienes toma las decisiones 
más trascendentales; teniendo como objetivo transformar la naturaleza humana y construir un hombre nuevo. $\mathrm{O}$ sea, los individuos tienen anuladas o restringidas ciertas libertades y pueden o deben comportarse de manera distinta a su condición natural libre, racional, individualista, etc.

Un conjunto de experiencias históricas de organización social -especialmente los sistemas socialistas- en las que el Estado fue o es el gran organizador de la economía y la sociedad están inspiradas en la idea del Estado amo.
Estado debe ser un servidor que, respetando la condición libre, racional y egoísta de la naturaleza humana, establece en afán optimizador, ciertos límites para evitar el accionar no paretiano, proveyéndole de servicios de seguridad, justicia, etc.; para garantizar el ejercicio de sus libertades y la certeza de sus decisiones y contratos. El origen del Estado tendría como objetivo la construcción de un hombre bueno.

La mayoría de países que se organizan en base al sistema democrático, han optado por conceptuar al Estado como un servidor. En la forma democrática actual, los individuos ejercen sus libertades y toman las grandes decisiones relacionadas con sus capacidades,

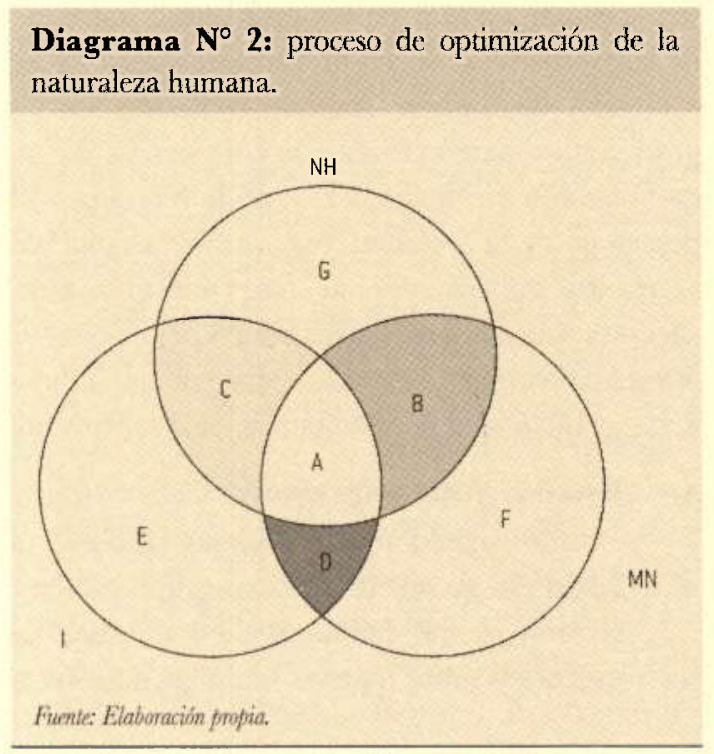

necesidades y aspiraciones en los marcos de la institucionalidad jurídico-política, establecida en la sociedad. En el ámbito de la economía, la denominada economía de mercado es la expresión de su libertad de elección; o sea libertad de consumo, producción e intercambio.

Hay que establecer, sin embargo, que las acciones optimizadoras que se logran con la formación moral y la creación del Estado no transforman ni cambian la naturaleza de los individuos, que está dada genéticamente. Tampoco se ha demostrado científicamente que estos optimizadores la alteren. La formación moral y la institucionalidad son hechos o acciones culturales y civilizadores, pero sin implicancia genética ni biológica.

\section{INTERACCIÓN DEL MARCO MORAL Y LA INSTITUCIONALIDAD EN EL PROCESO DE OPTIMIZACION DEL ACCIONAR INDIVIDUAL, SEGÚN SU NATURALEZA O CONDICIÓN HUMANA}

Para entender mejor este proceso, veamos el siguiente diagrama y sus lecciones.

El círculo $\mathbf{N H}$, Naturaleza Humana, representa el accionar no paretiano de la Naturaleza Humana, libre, racional, egoísta, etc. El círculo MM, Marco Moral, representa la educación del individuo en valores, buenas costumbres, etc. El círculo I Institucionalidad, el accionar del Estado a través de normas, instituciones y autoridades para validar solo el accionar paretiano de los individuos. $\boldsymbol{N H}, \boldsymbol{M M}$ e $\boldsymbol{I}$, son espacios infinitos, en expansión, como el universo mismo.

Las áreas $\boldsymbol{A}, \boldsymbol{B}$ y $\mathbf{C}$ simbolizan el accionar paretiano de los individuos gracias a la actuación del $\boldsymbol{M} \boldsymbol{M}$. y la $\boldsymbol{I}$. Cuanta mayor área de $\boldsymbol{N H}$ ocupen $\boldsymbol{A}, \boldsymbol{B}$ y $\boldsymbol{C}$, reduciendo $\boldsymbol{G}$ mayor será el comportamiento paretiano del individuo y su impacto en el desarrollo y progreso de la sociedad y viceversa.

El área $\boldsymbol{A}$ sería aquel espacio de accionar paretiano logrado a través de la conversión en normas legales, de preceptos morales, buenas costumbres o tradiciones ya existentes. $\boldsymbol{B}$, el espacio logrado por los individuos gracias a su formación ética, espiritual y observancia de buenas costumbres y tradiciones. Actualmente, debido

6. Filósofo inglés. (Wrington, Somerset, 1632; Oates, Essex, 1704). Exponente de un materialismo sensualista. Consideraba que la sociedad se basaba en un contrato y que el soberano debe obedecer las leyes; de otro modo la insurrección del pueblo es legítima. (Ibid.) 
a las crisis y transformaciones que enfrentan las redes clásicas formadoras de valores -familia, iglesia, educación y Estado- el aporte de $\boldsymbol{M} \boldsymbol{M}$ al accionar paretiano está perdiendo eficacia y espacio. Mientras $\boldsymbol{C}$ significa el espacio de accionar paretiano logrado gracias al establecimiento, desde el Estado, de ciertos límites y prohibiciones legales, obligando a los individuos por su observancia. La función de $\boldsymbol{I}$ en la obtención de mayores espacios de acción paretiana de los individuos es muy eficaz; asociado a la construcción y funcionamiento adecuado del Estado, sus instituciones y el establecimiento de buenas normas legales, en basc a un correcto sistema de incentivos. En esto radicarían las diferentes eficacias de $\boldsymbol{I}$ en las sociedades e individuos de los países desarrollados y en desarrollo. En cuanto a $\boldsymbol{D}$. representaría cl espacio potencial de normas morales, aún inobservadas por los individuos, traducibles en normas legales de observancia obligatoria. El área $\boldsymbol{E}$ personalizaría el potencial de normas legales aún inobservadas; ya sea por la incapacidad del Estado para imponerlas en la sociedad o porque -todavíano han sido aprobadas para su cumplimiento. El área $\boldsymbol{F}$ implicaría el potencial de normas morales y de buenas tradiciones aún inobservadas, por no haber sido inculcadas a los individuos. Finalmente, $G$ representa el accionar natural no paretiano que aun desarrollan los individuos y constituye el espacio por cubrir u optimizar con $\boldsymbol{M} \boldsymbol{M}$ e $\boldsymbol{I}$.

La posibilidad de que $\boldsymbol{N H}$. $\boldsymbol{M} \boldsymbol{M}$ e $\boldsymbol{I}$ se igualen significaría la promesa del proceso y accionar paretiano perfecto de la humanidad. Esto tal vez sea difícil de lograrlo dada la fuerza y gravedad del accionar natural, dada la escasez de recursos para lograrlo, especialmente tiempo, y dado el balance costo/beneficio que lo justifique. Aunque las áreas de accionar paretiano, $\boldsymbol{A}, \boldsymbol{B}$ y $\boldsymbol{C}$, en perspectiva se irán ampliando, nunca confluirán hasta hacer idénticos a $\boldsymbol{N H}, \boldsymbol{M} \boldsymbol{M}$. e $\boldsymbol{I}$. Este proceso infinito estará básicamente marcado por el carácter misterioso -aun desconocido- de muchas facetas de la naturaleza humana y la vida, y las adaptaciones de $\boldsymbol{M} \boldsymbol{M}$ e $\boldsymbol{I}$ que serán necesarias ante un mayor conocimiento de éstos.

\section{LA CIENGIA ECONÓMICA Y SU APORTE CIVILIZADOR}

Para que una ciencia sea considerada como tal, su definición y cuerpo teórico deben tener, entre otros, validez universal. En el caso de la Economía, su carácter universal se comprueba demostrando que para todo individuo es verdadero el principio de escasez, y dada la naturaleza humana, se hace imperativo que su asignación no sólo sea eficiente sino fundamentalmente, paretiana. La Economía es la ciencia de la eficiencia paretiana; por su objetividad, validez universal y el uso del método científico en su modelística y construcción teórica. La ciencia económica no se limita a estudiar el ámbito positivo de la conducta económica natural de los individuos; donde se observa un conjunto de asignaciones de recursos escasos de manera no paretiana; asociado a la presencia de fallos de mercado. En el ámbito normativo, la Ciencia Económica prescribe soluciones a tales fallos, para lo cual ha modelado teóricamente la situación ideal que garantiza asignaciones eficientes y paretianas, denominándose a tal Modelo como la situación de Mercado de Competencia Perfecta.

\section{El Modelo Teórico de Mercado de Competencia Perfecta}

El mecanismo que garantiza la asignación eficiente y paretiana de recursos escasos frente a opciones alternativas es el Mercado, en su acepción competitiva; a su vez es un gran ámbito de ejercicio de la naturaleza humana. Dado que la producción, el consumo y el intercambio son actividades humanas naturales es normal que sean también actividades de ejercicio de la libertad con resultados racionales. $\mathrm{O}$ sea, no son coercitivos y tienen resultados beneficiosos para los individuos, evaluados privadamente.

Toda situación de concurrencia de ofertantes y demandantes de un producto es denominada el Mercado del Producto. Todos los individuos son ofertantes en unos mercados y demandantes en otros. No existen demandantes ni ofertantes netos. Existe un mercado para cada bien o servicio. El mercado está conformado por dos fuerzas: la demanda y la oferta.

\section{a. El equilibrio del mercado competitivo}

El equilibrio del mercado es aquella situación en que la Demanda, $\boldsymbol{D}$, o intenciones de consumo y la Oferta, $\boldsymbol{S}$, o intenciones de producción, se equilibran en un nivel de $\boldsymbol{Q} \boldsymbol{o}$ unidades y a un determinado precio, Po; como se ve en el Gráfico 2.

\section{b. Las virtudes del Modelo Teórico de Mercado de Competencia Perfecta}

El Modelo encierra un conjunto de características y virtudes. La Curva de Demanda, $\boldsymbol{D}$, muestra las valoraciones 
Gráfico $N^{\circ}$ 2: equilibrio del mercado de competencia perfecta: virtudes del modelo.

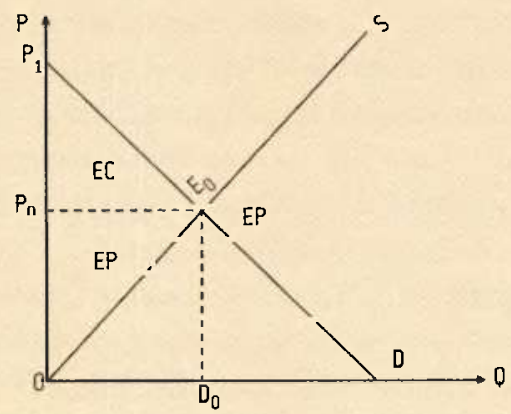

o disposición a pagar por las unidades consumidas; representa la conducta humana asociada al fenómeno del consumo de bienes o servicios; expresa con nitidez que los individuos valoran la escasez. La Curva de Oferta, $\boldsymbol{S}$, representa la valoración creciente de los recursos utilizados en la producción; muestra la disposición a cobrar por las cantidades producidas; representa la conducta humana asociada al fenómeno de la producción de bienes o servicios. El área $\mathbf{0 E o Q o}$ representa la eficiencia; es decir, los costos mínimos de producir $Q o$ unidades. El área $\boldsymbol{P I E o Q o O}$ representa el máximo valor o utilidad producida. Los triángulos $\boldsymbol{E} \boldsymbol{C}$, excedente del consumidor, y $\boldsymbol{E P}$, excedente del productor representan las ganancias o racionalidad en las decisiones de consumo, de producción y de intercambio. Po representa el precio correcto del bien y Qo es la producción socialmente óptima. $\boldsymbol{E} \boldsymbol{o}$ es el equilibrio Óptimo de Pareto. La economía, como la vida, es dinámica. No hay que imaginar un equilibrio de mercado como situación estática. En tanto las fuerzas de la Oferta y Demanda son funciones que contienen variables que las influyen, al cambiar una o algunas variables varían los equilibrios. Pero un nuevo equilibrio, analizado en estática comparativa, no niega sino vuelve a reproducir las virtudes del modelo.

\section{c. Supuestos del modelo.}

Los Modelos son representaciones simplificadas de la realidad. En los procesos de construcción o elaboración de teorías o conocimientos científicos, se acostumbra modelar los fenómenos en su versión correcta, natural, normal o lógica. Para llegar al establecimiento científico de modelos se hace necesario establecer ciertos supuestos, o sea, aquellas circunstancias que deben concurrir para el funcionamiento normal o adecuado de los fenómenos o realidades modeladas y teorizadas.

Para que funcione adecuadamente el Mercado de Competencia Perfecta y se logren todos los beneficios y virtudes, la Teoría Microeconómica ha establecido como necesarias la concurrencia de los siguientes supuestos: atomicidad, libre entrada y salida, producción homogénea, información perfecta y la inexistencia de externalidades. Mientras que los aspectos o condiciones - previamente existentes- que deben estar en la base de la sociedad, para que funcione perfectamente el mercado competitivo son la libertad, la propiedad privada, la estabilidad y las reglas de juego.

\section{d. La validez de los modelos.}

En los mercados reales no es factible verificar el funcionamiento del mercado en condiciones de competencia perfecta. Desde un ángulo epistemológico, sabemos que la validez de una Teoría o un Modelo no está determinada por el realismo de los supuestos, sino, en primer término, por su aplicabilidad para comparar, conocer, identificar y diagnosticar los problemas; y en segundo lugar por su utilidad para resolver o dar alternativas de solución a los problemas y hacer predicciones útiles. No se puede saber o conocer un problema sin compararlo con la situación normal o correcta que debe estar representada en un Modelo. Tampoco se puede resolver un problema sin conocer el Modelo, o sea la situación normal o correcta del problema o fenómeno. La construcción de los Modelos debe ser científica y no metafisica ni dogmática. El carácter normativo de las ciencias no se establece subjetivamente; se sustenta en el estado o situación de naturalidad, normalidad o perfección de un fenómeno, sistema estructura.

\section{INTERVENCIÓN DEL ESTADO Y EL PAPEL GIVILIZADOR DE LA ECONOMÍA}

En base al Modelo Teórico de Mercado de Competencia Perfecta, la Teoría Económica estudia y prescribe soluciones a todas aquellas circunstancias en que la economía real no esté 
organizada ni funcionando en forma eficiente, en el sentido de Pareto. Tales circunstancias son denominadas fallos de mercado y justifican la intervención del Estado en la Economía. Joseph Stiglitz, Premio Nobel de Economía del año 2001, ha logrado sistematizar un total de 8 Fallos de Mercado ${ }^{7}$. Las políticas públicas que ayudan a resolver los fallos de mercado tienen como eje orientador al Modelo Normativo de Mercado de Competencia Perfecta. La construcción de una institucionalidad económica competitiva, asociada

\section{BibLIOGRAFÍA}

\section{DÁvILA, Carlos Arturo.}

2008. Las Politicas Públicas como Instrumentos para resolver los fallos de mercado y el papel orientador del modelo Teónico de Competencia Perfecta. Arequipa, UNSA. Tesis Doctoral.

FRANK, Robert.

1992. Microeconomía y Conducta. Madrid, McGraw Hill.

FRIEDMAN, Milton \& ROSE.

1980. Libertad de Elegir. Barcelona, Ediciones Grijalbo, S.A.

FUKUYAMA, Francis.

1996. El Fin de la Historia y el Ultimo Hombre. Buenos Aires, Editorial Planeta S.A.

KAFKA, Folke.

1990. Análisis Económico. Lima Perú. CIUP. Séptima edición.

LAROUSSE

1996. Diccionario Enciclopédico.

MUÑ̃Z, Óscar.

1993. Después de las Privatizaciones: hacia el Estado Regulador. Santiago de Chile. Ediciones CIEPLAN. a la formación moral de los hombres, garantiza los resultados paretianos de la conducta económica natural de los individuos y grupos. Si en otras esferas de actuación humana, las consideradas como delitos por la moral o las leyes se sancionan legal y socialmente, en la economía los fallos de mercado $o$ asignaciones no paretianas se regulan, corrigen o internalizan a través de políticas públicas para garantizar que sus resultados sean paretianos ${ }^{8}$. Este es el ámbito normativo y el aporte civilizador de la Ciencia Económica.

PARODI T., Carlos.

1996. "El Estado regulador en el Perú". En: Revista Apuntes $\mathrm{N}^{\mathrm{o}}$ 40, I Semestre. Lima.

\section{STIGLITZ, Joseph.}

2003. La Economía del Sector Público. España, Antoni Bosch Editor.

\section{Recursos en linea:}

http://bilbaopundit.blogsome.com/2006/12/13/lanaturaleza.humana-no-es-un-mito/

http://www.eumed.net. Diccionario de Economía Política http://www.filosofia.org/cla/ari/azc03017.htm Tomás Várnagy. El pensamiento político de John Locke y el surgimiento del liberalismo. Capítulo II.

http://www.filosofia.org/cla/ari/azc03017.htm Origen del Estado y la sociedad.

http://www.laberinto.es Antonio Guerrero Torres y Moisés Vacaró Fernández. El origen del Estado.

http://www.html.rincondelvago.com/teoría-de-lanaturaleza-humana.html

7. 1. Fallos de la competencia. 2. Mercados incompletos. 3. Bienes públicos. 4. Externalidades. 5. Fallos de la información. 6. Inflación, desempleo y desequilibrios, cído económico. 7. Pobreza y Redistribución. 8. Bicnes preferentes. Los seis primeros muestran aquellas circunstancias en las que los mercados reales pueden no estar funcionando eficientemente en el sentido de Pareto, y los dos últimos muestran aquellas situaciones en las que, aun cuando los mercados sean eficientes en el sentido de Pareto, se amerita la intervención del Estado en la economía.

8. Las politicas públicas que relajan o debilitan la institucionalidad competitiva de la economía o distorsionan el funcionamiento de los mercados, se denominan Fallos del Estado. 